\title{
Effects of Microgravity on Human Physiology
}

\author{
Nguyen Nguyen, M.D. ${ }^{1,2}$, Gyutae Kim, Ph.D. ${ }^{1}$, Kyu-Sung Kim, M.D., Ph.D. ${ }^{1,2}$ \\ ${ }^{1}$ Research Institute for Aerospace and Medicine, Inha University, ${ }^{2}$ Department of Otolaryngology, Head and Neck \\ Surgery, College of Medicine, Inha University, Incheon, Korea
}

\author{
Received: March 2, 2020 \\ Revised: April 19, 2020 \\ Accepted: April 20, 2020 \\ Corresponding Author \\ Kyu-Sung Kim \\ Department of Otolaryngology, Head and Neck \\ Surgery, College of Medicine, Inha University, \\ 27 Inhang-ro, Jung-gu, Incheon 22332, Korea \\ Tel: $+82-32-890-2817$ \\ Fax: +82-32-890-3580 \\ E-mail: stedman@inha.ac.kr \\ ORCID \\ https://orcid.org/0000-0002-5650-3526
}

\begin{abstract}
Space exploration is one of the dreams of humankind. However, the intriguing environment was a challenge for the human body, where we must counter with many extreme conditions such as thermal support, radiation, microgravity. Life, as well as the human body, developed and evolved in the continuous presence of gravity, especially when living creatures transfer from the ocean to the land. Once this gravitational force doesn't impact on the body, the drastic changes occur. Some of these changes were observed immediately, while others progress only slowly. Since the first orbital flight was performed, several hazards for the organs of the human body were identified [1]. These changes in human physiology can reverse when astronauts return to Earth. This article will review the published findings of the effects of microgravity exposure on the human body.
\end{abstract}

Keywords: Microgravity, Aerospace medicine, Cardiovascular system, Musculoskeletal system, Neurovestibular system

\section{INTRODUCTION}

Physiology under extreme conditions reveals reactions of the human body, which may develop our knowledge of limits for a healthy individual. It may also help us to recognize premonitory signs of disease. Human physiology under microgravity (MG) is no exception. In April 1961, since Yuri Gagarin's first flight into space, it had become obvious that the environment of aerospace influences the human body in many ways. Even though humans can adapt to space, it can lead to problems when we return to Earth's gravity. The adaptation of humans can remain productive up to 1 year or longer. Many of the fundamental problems of aerospace medicine such as response to high altitudes, thermal support, dysbarism, hypoxia have been studied a long time before the human traveling aerospace by aviation and diving medicine. Aerospace, aviation, and diving medicine all involve operational changes, pressure excursions in body attitude and position, monitored breathing sources, and vital dependence on supportive mechanisms and life support equipment $[1,2]$. However, aerospace flight causes unique medical challenges. There was prolonged exposure to stressful stimuli such as acceleration forces, radiation, and MG. In particular, MG is a critical feature of aerospace flight.

To appreciate the uniqueness of aerospace conditions, we should consider that the protective atmosphere and the static gravitational field of $9.81 \mathrm{~m} / \mathrm{s}^{2}$ are major factors that made Earth amenable to life. Gravity force is a more or less stable factor of the environment. Since the dawn of life, thus, it has influenced the phylogenetic development of all organisms [3]. Gravity has been a major contributor to the evolution of Earth's organisms from the aquatic life to the terrestrial one. By forcing ancestral organisms to adapt to the new environment, the complex system for gravity sensing, fluid regulation, stability, and locomo- 
tion was required to involve the evolution. The changes in gravity caused the alternation of growth and remodeling in all tissues, and it also showed that these effects were exerted at the cell level [4]. In space, the changes of human physiology occur under microgravity conditions in which astronauts underwent rapid senescence in aerospace such as sleep, metabolic, endocrine disturbances, bone density loss, cardiovascular and hematic changes, muscle atrophy [1]. These symptoms, in fact, are similar in the way that humans get older [5]. However, the astronauts could recover when they returned to Earth. This review will focus on the effects of microgravity on the systems of the human body.

\section{EFFECTS ON THE CARDIOVASCULAR SYSTEM}

The mechanism of the changes in the cardiovascular system to MG is complex, and it did not completely understand. The cardiac function, peripheral vasculature, and autonomic nervous system could all play a role in control mechanisms [6,7]. Humans usually maintain in an upright position. Therefore, gravity will determine a pattern of blood distribution with $70 \%$ of the blood contained below the heart level in the human body [8]. When entering microgravity, blood and other fluids were no longer pulled down, and they would be redistributed as well as shifted to the torso and head (upper part of the body). The astronauts will show "chicken legs" and "puffy face" syndrome $[9,10]$. The redistribution of fluid toward the head caused the altered responses of baroreceptors, endocrine, and nervous systems [10].

The baroreceptors of central vasculature will trigger the suppression of the renin-angiotensin-aldosterone system. Its reflex was mediated by stretch-sensitive mechanoreceptors in the carotid sinuses, aortic arch, and atria. Also, it provided a negative feedback loop to regulate blood pressure. Subsequently, the system releases atrial natriuretic peptide, which leads to an increase in renal excretion of water and salt. Finally, the net reduction was observed in plasma volume [11]. The first 24 hours of aerospace flight were characterized by a $17 \%$ reduction of plasma volume, which would cause the transient increase of hematocrit level. Consequently, there led to a decrease in erythropoietin secretion and caused a reduction in the mass of erythrocytes [10]. The cardiac muscle is ready for different loading conditions. For example, left ventricular hypertrophy will commonly appear in diseases with increased afterload, such as aortic stenosis or chronic hypertension. It makes sense, therefore, that the cardiac muscle may also adapt to the changes in cardiovascular dynamics in MG. Some studies showed a reduction of the cardiac size after aerospace flight compared to pre-flight. The cardiac atrophy occurred after six weeks in horizontal bed rest stimulation, and after just ten days of the aerospace flight. A $12 \%$ reduction of left ventricular mass was revealed via magnetic resonance imaging [12]. The result obtained echocardiographic measurements of 17 crews before and following 5 to 8 days aerospace flight. A significant $28 \%$ reduction of stroke volume was immediately showed after landing, and it would recover to preflight values within two weeks [13].

\section{EFFECTS ON THE MUSCULOSKELETAL SYSTEM}

The full strength of the muscular and skeletal system is essential to support an upright posture. There was a balance between bone resorption and bone formation. However, in MG, the astronauts no longer need them fully, and MG induced uncoupling of bone transformation between resorption and formation. Therefore, prolonged exposure to MG would result in bone demineralization and muscle atrophy $[14,15]$. Bone demineralization began immediately on arrival in aerospace. The urinary and fecal excretion of calcium increased during the first day in space, and it continued throughout the mission $[10,16]$. In turn, the increase in the excretion of calcium may affect various organs, specifically the kidney. For example, as shown in the previous study, the risk of renal stone formation would be increased, and it could lead to serious consequences during aerospace flight [17]. In addition, the changes in the site of blood-forming cells (bone marrow) were also linked to bone loss. In reference to bone metabolism, some studies have investigated the markers and the levels of vitamin D, bALP, parathyroid hormone (PTH), osteocalcin, calcitonin, and $\mathrm{Ca}^{++}$. A significant decrease of $1,25(\mathrm{OH})_{2}$-vitamin $\mathrm{D}$ and its precursor was realized by results from the Mir18 mission, while calcitonin levels were unchanged both in aerospace and back on Earth [18]. The PTH level rapidly increased when the astronauts returned to Earth. There indicated that the det- 
rimental mass bone was restored because PTH promoted bone resorption as well as elevated blood $\mathrm{Ca}^{++}$levels via activating osteoclasts. Moreover, PTH also acted on the kidney to decrease $\mathrm{PO}_{4}^{3-}$ and increase $\mathrm{Ca}^{++}$reabsorption [19]. Those suggested that the cause of bone loss in MG may mainly involve the impairment of vitamin D production and kidney function rather than to the significant hormone imbalance (calcitonin, PTH). Therefore, the reduced vitamin $\mathrm{D}$ level may induce a decrease of $\mathrm{Ca}^{++}$ fixation in bone mass and reabsorption in kidneys. Consequently, the $\mathrm{Ca}^{++}$excretion was increased despite the normal levels of the hormone [18].

The most muscles in the body were used as anti-gravity muscles. Their main function was to maintain our body upright in the gravitational environment and induce locomotion. They would mostly be affected by MG. Muscles lose both strength and mass during aerospace flight. The fundamental cause of the muscle atrophy was the absence of the gravity which loaded on bone and muscle on Earth. The grade of muscle atrophy depended on the different duration of muscle unloading and the muscle size before flight [20]. For example, as compared to the knee extensors, atrophy of the plantar flexors was more pronounced. There was possibly explained by higher load stress of the plantar flexors than one of the knee extensors. Thus, the former would cause more damage than the latter during uploading in space. The damage not only derives from fiber loss but also the reduction in their protein synthesis and size [1]. Protein degradation in muscle fiber increased while protein synthesis decreased.

\section{EFFECTS ON THE NEUROVESTIBULAR SYSTEM}

In MG conditions, the central nervous system (CNS) must be reprogrammed to adapt to new neural information and the new movement possibilities. There leads to a new level of disorientation within the first hours or a few days in the MG environment. Thus, most astronauts experienced symptoms of neurovestibular acclimation during those days. A similar period of re-acclimation was also observed when the astronauts returned to Earth. The predominant symptoms included cold sweating, facial pallor, stomach awareness, nausea, and vomiting. This syndrome has been termed as space motion sickness (SMS) [21]. The SMS was not reported during Mercury and Gemini flight while it was observed in the Apollo and Skylab missions [22]. This difference can be explained by the limited opportunity for movement. With larger capsules like in the Russian space program or in the Apollo and Skylab missions, the increasing movement of the astronauts would cause the conflicting motor, visual, and vestibular input. Two hypotheses were proposed for the causes of the SMS: the "fluid shift" and the "sensory conflict" hypothesis. The fluid shift mechanism suggested that the SMS was induced by the headward shifting of the body fluids. When the astronauts entered in MG environment, the hydrostatic pressure was reduced in the lower part of the body. The decreased hydrostatic load induced the shifting of the fluid from the lower to the upper part of the body. There resulted in a decrease of the leg volume as well as the thinned leg looking like "bird legs." On the other hand, the headward fluid shift leads to the concomitant changes in the cerebrospinal-fluid column, intracranial pressure, or the inner ear fluid pressure. The changed pressure would alter the response properties of vestibular hair cells and inducing SMS [22]. However, this hypothesis has been largely debated. The SMS symptoms were not reported during the limited mobility in early aerospace flight, and they did not occur in the headward fluid shift models (head-down tilt bed rest) [23]. Sensory conflict theory is now widely accepted, and there is the primary cause of the SMS [22]. The human orientation in 3D space, under normal gravitational conditions, was based on the sensory information from vestibular end organs, the visual, haptic, and proprioceptive senses. If the expected information and actual sensory inputs were mismatched or not compatible, the motion sickness would appear. The CNS integrated the information from otoliths (linear acceleration and gravity) and semicircular canals (angular acceleration) to stabilize eye and body movement as well as maintain spatial orientation. In aerospace, yet, the information from vestibular end organs that was transmitted to the CNS was inappropriate. Consequently, head movements in the MG environment, especially in the roll and pitch planes, probably were the dominant stimuli that induced the SMS [24].

\section{CONCLUSION}

Humans adapt to the aerospace environment, which was characterized by some altered conditions, especially 
the absence of gravity, through cardiovascular adaptation as well as drastic changes in bone density, muscle integrity, and vestibular information. A good understanding of the disorders associated with aerospace flight will help clinicians to refine the delivery of medical care for the astronauts. The observation of muscle atrophy and bone demineralization in the astronauts was considered to support the idea that some organ systems may age faster in MG conditions. Therefore, aging most likely affects the organs of the human body, similarly to exposure to MG. These changes allowed scientists to consider that the astronauts probably were an appropriate model for investigating the mechanism of aging.

\section{ACKNOWLEDGEMENTS}

This research was supported by Basic Science Research Program through the National Research Foundation of Korea (NRF) funded by the Ministry of Education (2018R1A6A1A03025523).

\section{CONFLICTS OF INTEREST}

No potential conflict of interest relevant to this article was reported.

\section{ORCID}

Nguyen Nguyen, https://orcid.org/0000-0003-1878-394X Gyutae Kim, https://orcid.org/0000-0001-6747-8189 Kyu-Sung Kim, https://orcid.org/0000-0002-5650-3526

\section{REFERENCES}

1. Demontis GC, Germani MM, Caiani EG, Barravecchia I, Passino C, Angeloni D. Human Pathophysiological Adaptations to the Space Environment. Frontiers in physiology 2017:8:547-547.

2. Barratt M. Chapter 1: Physical and bioenvironmental aspects of human space flight, in Principles of Clinical Medicine for Space Flight, Springer 2008. p. 3-26.

3. Anken RH, Rahmann H. Gravitational Zoology: How Animals Use and Cope with Gravity, in Astrobiology: The Quest for the Conditions of Life, G. Horneck and C. BaumstarkKhan, Editors, Springer Berlin Heidelberg: Berlin, Heidelberg. 2002. p. 315-333.

4. Ingber DE. Tensegrity: the architectural basis of cellular mechanotransduction. Annual Review of Physiology 1997;59(1):575-599.
5. Vernikos J, Schneider VS. Space, Gravity and the Physiology of Aging: Parallel or Convergent Disciplines? A MiniReview. Gerontology 2010;56(2):157-166.

6. Levine BD, Zuckerman JH, Pawelczyk JA. Cardiac Atrophy After Bed-Rest Deconditioning. Circulation 1997;96(2):517525.

7. Levine BD, Pawelczyk JA, Ertl AC, Cox JF, Zuckerman JH, Diedrich A, et al. Human muscle sympathetic neural and haemodynamic responses to tilt following spaceflight. The Journal of Physiology 2002;538(1):331-340.

8. Antonutto G, Di Prampero PE. Cardiovascular deconditioning in microgravity: some possible countermeasures. European Journal of Applied Physiology 2003;90(3):283-291.

9. Aubert AE, Beckers F, Verheyden B. Cardiovascular function and basics of physiology in microgravity. Acta Cardiologica 2005;60(2):129-151.

10. Williams D, Kuipers A, Mukai C, Thirsk R. Acclimation during space flight: effects on human physiology. CMAJ : Canadian Medical Association journal = journal de l'Association medicale canadienne 2009;180(13):1317-1323.

11. Shen M, Frishman WH. Effects of Spaceflight on Cardiovascular Physiology and Health. J Cardiology 2019;27(3):122126.

12. Perhonen MA, Franco F, Lane LD, Buckey JC, Blomqvist CG, Zerwekh JE, et al. Cardiac atrophy after bed rest and spaceflight. Journal of Applied Physiology 2001;91(2):645-653.

13. Bungo MW, Goldwater DJ, Popp RL, Sandler H. Echocardiographic evaluation of space shuttle crewmembers. Journal of Applied Physiology 1987;62(1):278-283.

14. Carmeliet G, Bouillon R. The effect of microgravity on morphology and gene expression of osteoblasts in vitro. The FASEB Journal 1999;13(9001):129-134.

15. Hargens AR, Bhattacharya R, Schneider SM. Space physiology VI: exercise, artificial gravity, and countermeasure development for prolonged space flight. European Journal of Applied Physiology 2013;113(9):2183-2192.

16. Clément G, The Musculo-Skeletal System in Space, in Fundamentals of Space Medicine, Springer New York: New York, NY. 2011. p. 181-216.

17. Whitson PA, Pietrzyk RA, Morukov BV, Sams CF. The Risk of Renal Stone Formation during and after Long Duration Space Flight. Nephron 2001;89(3):264-270.

18. Smith SM, Wastney ME, Morukov BV, Larina IM, Nyquist LE, Abrams SA, et al. Calcium metabolism before, during, and after a 3-mo spaceflight: kinetic and biochemical changes. American Journal of Physiology-Regulatory, Integrative and Comparative Physiology 1999;277(1):1-10.

19. Lee SMC, Schneider SM, Boda WL, Watenpaugh DE, Macias BR, Meyer RS, et al. LBNP exercise protects aerobic capacity and sprint speed of female twins during 30 days of bed rest. Journal of Applied Physiology 2009;106(3):919-928.

20. Belavý DL, Miokovic T, Armbrecht G, Richardson CA, Rittweger J, Felsenberg D. Differential atrophy of the lowerlimb musculature during prolonged bed-rest. European Journal of Applied Physiology 2009;107(4):489. 
21. Heer M, Paloski WH. Space motion sickness: Incidence, etiology, and countermeasures. Autonomic Neuroscience 2006;129(1):77-79.

22. Reschke MF, Bloomberg JJ, Harm DL, Paloski WH, Layne C, Mcdonald V. Posture, locomotion, spatial orientation, and motion sickness as a function of space flight. Brain Research Reviews 1998;28(1):102-117.
23. Oman A, Charles M. Sensory conflict theory and space sickness: our changing perspective. Journal of vestibular research : equilibrium \& orientation 1998;8(1):51-56.

24. Young LR, Sinha P. Spaceflight influences on ocular counterrolling and other neurovestibular reactions. OtolaryngologyHead and Neck Surgery 1998;118(3, Supplement):31-34. 